Research, Society and Development, v. 9, n. 8, e504985844, 2020

(CC BY 4.0) | ISSN 2525-3409 | DOI: http://dx.doi.org/10.33448/rsd-v9i8.5844

\title{
Medicina Veterinária e a Aprendizagem Baseada em Problemas (ABP): uma experiência aplicada às disciplinas de Microbiologia e Imunologia
}

Veterinary Medicine and Problem-Based Learning (PBL): an experience applied to the disciplines of Microbiology and Immunology

\section{Medicina Veterinaria y Aprendizaje Basado en Problemas (ABP): una experiencia aplicada a las disciplinas de Microbiología e Inmunología.}

Recebido: 20/06/2020 | Revisado: 30/06/2020 | Aceito: 02/07/2020 | Publicado: 15/07/2020

\section{Renata Ferreira dos Santos}

ORCID: https://orcid.org/0000-0002-1033-275X

Centro Universitário do Vale do Araguaia, Brasil

E-mail: renatafdsantos@hotmail.com.br

Jordana Belos dos Santos

ORCID: https://orcid.org/0000-0002-7822-4106

Centro Universitário do Vale do Araguaia, Brasil

E-mail: jordana@univar.edu.br

Cristina Alves Moreira

ORCID: https://orcid.org/0000-0003-0077-9037

Centro Universitário do Vale do Araguaia, Brasil

E-mail: cristinaalvesmoreira50bg@gmail.com

\section{Resumo}

A Aprendizagem Baseada em Problemas (ABP) é uma metodologia de ensino e aprendizagem que utiliza problemas do cotidiano profissional de diferentes áreas, para motivar a aprendizagem para a futura atuação dos acadêmicos como profissionais e cidadãos. Assim, na perspectiva de possibilitar aos alunos do Curso de Medicina Veterinária a compreensão das relações entre os conteúdos de Microbiologia e Imunologia Veterinária, após algumas reflexões surgiu a necessidade de atividades interdisciplinares. Nesse sentido, o objetivo do presente trabalho é apresentar um estudo de caso sobre emprego do método ABP como metodologia ativa no curso de Medicina Veterinária. Para isso, uma situação problema foi elaborada, relacionando os diagnósticos imunológicos, que são comuns na prática clínica veterinária e as características do agente etiológico em questão, no caso as bactérias do gênero 
Brucella spp, causadora de perdas produtivas em grandes animais. Observou-se que as atividades práticas e a metodologia ativa contribuíram para que os alunos conseguissem responder a solução-problema que, a princípio, foi vista por eles como um enigma e ao mesmo tempo como um desafio na busca de uma solução. Assim, sugere-se que tal atividade seja aplicada em diferentes disciplinas, com a função de desenvolver a autonomia dos discentes frente às adversidades que os mesmos poderão deparar na vida profissional.

Palavras-chave: Metodologia ativa; Ciências agrárias; Diagnóstico imunológico; Ensino de veterinária; Ensino.

\begin{abstract}
Problem-Based Learning (PBL) is a teaching and learning methodology that uses everyday professional problems from different areas, to motivate learning for the future performance of academics as professionals and citizens. Thus, in order to enable students of the Veterinary Medicine Course to understand the relationships between the contents of Microbiology and Veterinary Immunology, after some reflections, the need for interdisciplinary activities arose. In this sense, the objective of the present work is to present a case study on the use of the ABP method as an active methodology in the Veterinary Medicine course. For this, a problem situation was elaborated, relating the immunological diagnoses, which are common in veterinary clinical practice and the characteristics of the etiological agent in question, in this case bacteria of the genus Brucella spp, which causes productive losses in large animals. It was observed that the practical activities and the active methodology contributed for the students to be able to answer the problem solution that, at first, was seen by them as an enigma and at the same time as a challenge in the search for a solution. Thus, it is suggested that such activity be applied in different disciplines, with the function of developing the students' autonomy in the face of the adversities that they may face in their professional life.
\end{abstract}

Keywords: Active methodology; Agrarian sciences; Immunology diagnosis; Veterinary teaching; Teaching.

\title{
Resumen
}

El Aprendizaje basado en problemas (ABP) es una metodología de enseñanza y aprendizaje que utiliza problemas profesionales cotidianos de diferentes áreas, para motivar el aprendizaje para el futuro desempeño de académicos como profesionales y ciudadanos. Por lo tanto, para permitir a los estudiantes del Curso de Medicina Veterinaria comprender las relaciones entre los contenidos de Microbiología e Inmunología Veterinaria, después de algunas reflexiones, 
surgió la necesidad de actividades interdisciplinarias. En este sentido, el objetivo del presente trabajo es presentar un estudio de caso sobre el uso del método ABP como metodología activa en el curso de Medicina Veterinaria. Para esto, se elaboró una situación problemática, relacionando los diagnósticos inmunológicos, que son comunes en la práctica clínica veterinaria y las características del agente etiológico en cuestión, en este caso bacterias del género Brucella spp, que causan pérdidas productivas en animales grandes. Se observó que las actividades prácticas y la metodología activa contribuyeron a que los estudiantes pudieran responder a la solución del problema que, al principio, vieron como un enigma y al mismo tiempo como un desafío en la búsqueda de una solución. Por lo tanto, se sugiere que dicha actividad se aplique en diferentes disciplinas, con la función de desarrollar la autonomía de los estudiantes frente a las adversidades que puedan enfrentar en su vida profesional.

Palabras clave: Metodología activa; Ciencias agrarias; Diagnóstico inmunológico; Enseñanza en veterinária; Enseñanza.

\section{Introdução}

Atualmente, muito se tem discutido em relação às novas metodologias de ensino, como sala de aula invertida, aprendizagem baseada em problemas, o ensino como pesquisa, aula expositiva, estudo de texto, discussão e debate e seminário.

Nesse sentido, a metodologia conhecida como Problem Based Learning (PBL) ou Aprendizagem Baseada em Problemas (ABP) tem sido considerada atualmente como um dos métodos pedagógicos mais atrativos em cursos do ensino superior, permitindo ao estudante ser sujeito ativo na construção do seu próprio conhecimento. Tal método, estimula a gestão participativa dos protagonistas da experiência, estudantes e docentes e a reorganização da relação entre a teoria e a prática (Cyrino \& Toralles-Pereira, 2004).

Com origem na Escola de Medicina da Universidade McMaster, Canadá, na década de 1960, o método utiliza-se de problemas do cotidiano (reais ou simulados) para iniciar, enfocar e motivar a aprendizagem de teorias, habilidades e atitudes. Nesse sentido, o ensinoaprendizado fica pautado na construção do conhecimento em vez de simplesmente memorizado e acumulado (Escrivão Filho \& Ribeiro, 2009).

A metodologia de aprendizagem ativa tem sido largamente utilizada em cursos da área da saúde: Medicina (Bento et al., 2017), Odontologia (Saliba et al., 2008), Enfermagem (Faustino, 2013), e em alguns cursos das áreas das exatas (Escrivão Filho \& Ribeiro, 2009), além de alguns cursos das áreas das ciências sociais. 
Apesar das experiências mencionadas, a utilização de metodologias ativas em cursos de graduação em Medicina Veterinária no Brasil ainda é pouco relatada, principalmente se considerarmos que existem mais de 280 instituições de ensino superior (IES) no país que oferecem esse curso em seus programas de graduação.

O Brasil conta com um terço das escolas de Medicina Veterinária do mundo. E apesar disso, apenas alguns autores relataram a utilização do método ABP no ensino de Medicina Veterinária aplicado à disciplina de Química (Lucena; Souza; Campos, 2012), Biologia Celular e a Clínica Médica Veterinária (Conrado et al., 2017).

A profissão é responsável por áreas estratégicas na economia, saúde e sustentabilidade e sua base está na formação profissional (CFMV, 2012). As atuais Diretrizes Curriculares Nacionais (DCN) da educação universitária apontam para a necessidade de implementação de novos modos de pensar e praticar o processo de ensino-aprendizagem. A Resolução CNE/CES $n^{\circ}$ 1/2003 define os princípios, fundamentos, condições e procedimentos da formação de médicos veterinários (Brasil, 2003).

No que diz respeito ao perfil profissional do Médico Veterinário, as Diretrizes Curriculares Nacionais descrevem o perfil do formando egresso/profissional, com formação generalista, humanista, crítica e reflexiva, apto a compreender e traduzir as necessidades de indivíduos, grupos sociais e comunidades, com relação às atividades inerentes ao exercício profissional (Ibdi, 2003).

Desse modo, o Conselho Federal de Medicina Veterinária (CFMV), publicou no ano de 2012, o documento intitulado "Estratégias de ensino aprendizagem para desenvolvimento de competências humanísticas: propostas para formar Médicos Veterinários para um mundo melhor", com objetivo de propor técnicas e ferramentas de ensino capazes de auxiliar que docentes e IES alcancem patamares mais elevados de excelência no ensino e na formação de Médicos Veterinários (CFMV, 2012). A publicação do documento traz uma reflexão sobre os valores da educação e vai além, com sugestão de técnicas para aqueles que têm o comprometimento com a formação dos futuros profissionais.

Entre as grandes vantagens da utilização do ABP estão: melhor compreensão dos temas estudados e melhor retenção dos conhecimentos pelos alunos, facilidade de troca de conhecimentos e habilidades aprendidos em classe para o mundo profissional, no método o estudante é responsável pela própria aprendizagem, desenvolvimento de habilidades interpessoais e do espírito de equipe, melhorando o relacionamento entre estudantes, e possibilidade de envolvimento interdisciplinar (Gôuvea \& Fonseca, 2017). 
Nesse sentido, o objetivo do presente trabalho é apresentar um estudo de caso sobre emprego do método ABP como metodologia ativa no curso de Medicina Veterinária., além de analisar as percepções dos estudantes de Medicina Veterinária, do segundo ano de Medicina Veterinária do Centro Universitário do Vale do Araguaia (UNIVAR), para posteriores aplicações em diversas disciplinas do curso.

\section{Metodologia}

Neste estudo realizou-se uma pesquisa qualitativa, na modalidade estudo de caso (Gouvêa et al., 2016). Trata-se de um tipo de pesquisa qualitativa, na qual o pesquisador procura estudar um fenômeno único de modo detalhado (Pereira et al., 2018). Foram 51 estudantes, do segundo ano, de Medicina Veterinária do Centro Universitário do Vale do Araguaia (UNIVAR), os envolvidos nesse estudo.

Em relação às disciplinas, foram selecionadas aquelas pertencentes à grade curricular do ano, que apesar de serem do ciclo básico, são importantes para preparar o aluno para as diversas áreas da Medicina Veterinária, tais como Clínica de Pequenos e Grandes Animais, Inspeção e Tecnologia de Produtos de Origem Animal, Zoonoses e Saúde Pública, Doenças Infecciosas e Parasitárias dos Animais Domésticos, entre outras. Uma vez interligadas, podese ajudar os acadêmicos a selecionar e sintetizar informações de forma a escolher os procedimentos de avaliação e intervenção mais adequados para cada situação.

Para realização da atividade três etapas foram utilizadas para resolução da situaçãoproblema. As docentes das disciplinas de Microbiologia e Imunologia foram as responsáveis pela tutoria nos encontros.

$\mathrm{Na}$ etapa inicial, os grupos foram distribuídos e os alunos deveriam diagnosticar um bovino com suspeita de brucelose, escolhendo os testes e quais materiais biológicos deveriam ser colhidos. A segunda etapa consistiu na fundamentação teórica do problema previamente exposto. Dessa forma, os mesmos, distribuídos em grupos, elaboraram o protocolo para realização dos testes que poderiam ser utilizados para o diagnóstico de triagem para Brucella spp.

Para tanto, buscaram informações sobre as características do agente etiológico da doença, quais os testes preconizados pelo Ministério da Agricultura, Pecuária e Abastecimento (MAPA) para o diagnóstico da doença e quais os tipos de reação ocorreriam para fazer a leitura dos exames. 
Desta forma, os grupos elaboraram o roteiro para execução dos testes, que foi discutido em sala de aula, no qual os mesmos abordaram sobre os pontos a serem realizados durante a realização dos testes. Os docentes envolvidos na atividade orientaram sobre os materiais que estavam faltando e o que poderia ser melhorado para chegar ao diagnóstico final da doença.

A terceira etapa consistiu na realização dos testes no laboratório, no qual os protocolos previamente descritos pelos discentes foram utilizados para realização do Teste do Antígeno Acidificado Tamponado (AAT) e Teste de Anel em Leite (TAL) para o diagnóstico.

Ao final, os discentes elaboraram o relatório, fazendo uma reflexão sobre o seu aprendizado e relatando os novos conhecimentos e sua aplicabilidade no caso em questão.

\section{Resultados e Discussão}

Inicialmente, os alunos demonstraram dificuldades com a nova metodologia, por estarem acostumados com a metodologia tradicional, de aulas expositivas e centrada no professor. Escrivão Filho \& Ribeiro (2009), discutiram as deficiências do modelo tradicional na formação profissional, uma vez que, em sua maioria, os discentes demonstram desinteresse e apatia.

Pimpão et al. (2017) relatam a dificuldade atual dos professores universitários obterem a atenção do estudante em sala de aula, é necessário ser inovador na forma de ensinar, devido as facilidades de acesso as informações juntamente com o mundo tecnológico, tornando-se um desafio para toda a comunidade acadêmica.

Contudo, mesmo com a dificuldade e resistência inicial, assim como em outras investigações, os discentes participantes deste estudo reconheceram, na sua maioria, que a aprendizagem baseada em problemas trouxe contribuições importantes no que se refere ao aprendizado aplicado à prática profissional.

Por meio dos relatórios elaborados ao término da atividade foi possível verificar as seguintes opiniões dos discentes em relação à atividade:

"Os métodos aplicados em sala de aula e laboratório caracterizam-se como complemento essencial para a formação e consolidação do saber acadêmico, pois é a oportunidade que o mesmo tem de visualizar de forma detalhada e tateável, além de aprofundar os estudos em novos conteúdos baseados em sua própria conclusão, pela dinâmica da atividade. ", 
Analisando-se o enunciado em seu discurso, observa-se que o declarante possui algum saber acadêmico e noção da questão dos conteúdos necessários para o aprendizado do futuro médico veterinário e isso é um bom sinal de que ele já está inserido nesse contexto e meio da área em foco neste artigo e, além disso, verifica-se que a proposta de trabalhar casos clínicos para o aprendizado da Microbiologia e Imunologia possibilita o aluno aplicar o complexo conhecimento adquirido em sala de aula rapidamente, pois sem estes muitas vezes o conteúdo torna-se de difícil fixação e aplicação.

Segundo Lanne (2008), pelo método tradicional, muitas vezes os estudantes são incapazes de efetivamente raciocinar e a aplicar o conhecimento para situações da vida real quando obtido pelos métodos tradicionais de educação.

Ainda em relação aos depoimentos, outros discentes participantes da atividade afirmaram que:

"A atividade que compreende os conteúdos de Microbiologia e Imunologia Veterinária, atingiu seus objetivos em como diagnosticar a brucelose. Auxiliando no aprendizado que será necessário para os anos seguintes do curso e para a vida profissional do veterinário. "

Verifica-se por meio dos enunciados que os alunos se mostram comprometidos e envolvidos na questão do aprendizado e tudo indica que esse fenômeno está relacionado ao tipo de atividade que leva os estudantes a se envolverem com problemas reais e a assumir a responsabilidade pelo seu aprendizado. Os próprios discentes relataram e enfatizaram sobre essa importância de apesar de se tratar de disciplinas do ciclo básico, a atividade que poderá ser aplicada nas disciplinas dos próximos anos e consequentemente na formação profissional. Ainda afirmaram que:

"Os métodos aplicados em aula prática foi e é de extrema importância para a vida profissional, aprimorando nossos conhecimentos e aprendendo como proceder $e$ desenvolver o diagnóstico exato da brucelose bovina."

Assim, em relação à temática escolhida, a microbiologia associada à imunologia, com foco no gênero Brucella e seus métodos diagnósticos, observou-se que os resultados foram satisfatórios. Da mesma forma, alguns autores enfatizaram essa importância da aplicação de novos métodos associados às disciplinas de Microbiologia e Imunologia. 
Paulo et al. (2012) \& Câmara et al. (2017) afirmaram em seus estudos que as aulas de imunologia práticas e teóricas são desenvolvidas para que o aluno possa sedimentar seu aprendizado e dessa forma, um importante recurso que contribui de modo significativo ao processo de aprendizagem dos alunos em relação à disciplina é a Aprendizagem Baseada em Problemas (ABP).

No que se refere a microbiologia, Bravo \& Paixão (2013) revelaram que a metodologia PBL é um bom instrumento para ensino da Microbiologia, atraindo a atenção do discente e permitindo-lhe o auto aprendizado motivado. Verifica-se que, a discussão dos casos clínicos demonstra ser um diferencial para o sucesso do aprendizado no qual o conhecimento técnico transcende os livros e se incorpora às atividades práticas inerentes à profissão.

Vale ressaltar que os conteúdos abordados no âmbito das disciplinas não sofreram alteração em virtude da adoção da nova estratégia de ensino-aprendizagem. Uma vez, que em anos anteriores o conteúdo era abordado de forma expositiva, tanto na disciplina de Imunologia quanto na disciplina de Microbiologia, e em relação à aula prática os roteiros eram elaborados pelos docentes e previamente entregues aos discentes no momento da realização dos testes, sem o levantamento crítico sobre o que poderia ser utilizado.

A promoção do potencial de pensamento crítico foi desenvolvida através de processos de reflexão no encontro com a realidade do caso clínico simulado, uma vez que quando indagados sobre os materiais a ser utilizados e se colhido de forma errônea ou incompleta os mesmos não chegariam ao resultado dos testes. Ajudando-os a crescer e a estar melhor preparado para o desempenho profissional.

Assim, desta forma o conteúdo, trabalhado de uma maneira diferente pelo professor, produziu resultados de aprendizagem diferentes. Além disso, observou-se que a aplicação desta metodologia permitiu o desenvolvimento do pensamento crítico, analítico e reflexivo nos discentes, competências consideradas fundamentais, sendo uma das metas propostas pelo Conselho Federal de Medicina Veterinária. Possibilitando que os discentes e futuros profissionais, se tornem cada vez mais autónomos na interpretação e resolução de problemas os quais são normais de surgirem ao longo de suas carreiras e vidas profissionais.

A experiência da posição de tutora foi um tanto quanto diferente para as docentes envolvidas, uma vez que a formação das mesmas ocorreu na forma tradicional. Porém se verifica a importância em relação à introdução de novas metodologias de ensino, uma vez que as gerações são diferentes e as necessidades também. 


\section{Considerações Finais}

A experiência com o uso do PBL nas disciplinas de Microbiologia e Imunologia Veterinária foi uma oportunidade enriquecedora, tanto para os estudantes de graduação em Medicina Veterinária do Centro Universitário do Vale do Araguaia (Univar), quanto para professoras/tutoras. As respostas dos alunos indicam que eles foram mais ativos no processo de aprendizagem, consideraram que o aprendizado ficou mais contextualizado e que a metodologia PBL facilitou a compreensão do processo de raciocínio clínico e sua melhor aplicabilidade para os anos posteriores e para vida profissional. Dessa forma, sugere-se que tal atividade seja mais aplicada para diferentes disciplinas, com a função de desenvolver a autonomia dos discentes frente as adversidades que os mesmos poderão deparar na vida profissional.

\section{Referências}

Bento, L. M. A. (2017). Percepção dos Alunos de Medicina Quanto a Aprendizagem X Ansiedade na Metodologia Ativa. Revista de Ensino, Educação e Ciências Humanas, 18 (2): 178-182.

Brasil. (2003). Resolução no 1/03 - Conselho Nacional de Educação. Câmara de Educação Superior. Diretrizes Curriculares Nacionais dos Cursos de Graduação em Medicina Veterinária. Diário Oficial da União. Brasília, 37(1): 15-6, fev. 2003. Disponível em: $<$ http://portal.mec.gov.br/cne/arquivos/pdf/ces012003.pdf >.

Bravo, L. G., \& Paixão, G. C. A. (2013). Discussão de casos clínicos microbianos por meio da ferramenta Prezi: aprendizagem baseada em problemas aliada ao uso de novas mídias audiovisuais. Revista Diálogos Acadêmicos, 2 (1): 48-55.

Câmara, L. M. C., Castro, I. H. R., Férrer, D. P. C., Mello, I. L., Queiroz, J. Á. N., \& Freitas, M. V. C. (2007). A aprendizagem colaborativa no ensino da imunologia no curso de enfermagem da Universidade Federal do Ceará. Olho Mágico, 14 (2): 48. 
Cyrino, E. G., Toralles-Pereira, M. L. (2004). Trabalhando com estratégias de ensinoaprendizado por descoberta na área da saúde: a problematização e a aprendizagem baseada em problemas. Cadernos de Saúde Pública, 20 (3): 780-788.

CFMV. (2012). Estratégias de ensino aprendizagem para desenvolvimento de competências humanísticas: Propostas para formar médicos veterinários para um mundo melhor. Brasília: CFMV, 2012. Disponível em: <http://portal.cfmv.gov.br/uploads/files/ Estrategias\%20de\%20Ensino-aprendizagem\%20para\%20Desenvolvimento\%20das\%20 Competencias\%20Humanisticas_site.pdf.>.Acesso em: 31 maio de 2018.

Conrado, A. L. V., Borges, R. G., Ramos, C. C., Cardoso, F., Terplak, R. (2017). Plano de trabalho docente integrativo entre a biologia celular e a clínica médica veterinária no modelo de PBL misto. Revista Brasileira de Ensino Superior, 3 (2):18-37.

Escrivão Filho, E. \& Ribeiro, L. R. C. (2009). Aprendendo com PBL- Aprendizagem Baseada em problemas: relato de uma experiência em cursos de engenharia da EESC-USP. Minerva, 6 (1): 23-30.

Faustino, A. M. (2013). Aplicação da Aprendizagem baseada em problemas na Graduação de Enfermagem. Revista Eletrônica Gestão \& Saúde, 4 (1): 1570-1581.

Gouvêa, E. P., Odagima, A. M., Shitsuka, D. M. (2016). Metodologia ativa: um estudo de caso sobre a ferramenta glossário em ambientes virtuais de educação a distância. Educação, Gestão e Sociedade: Revista da Faculdade Eça de Queirós, 6 (22), 1-13.

Gouvêa, G., \& Fonseca, R. F. (2017). Reflexões acerca do uso da aprendizagem baseada em problemas no ensino de conceitos matemáticos. Trilhas Pedagógicas, 7 (7),123-144.

Paulo, S. P., Silva, P. O., Coutinho, T. E. B., \& Santos, P. O. M. (2012). Atividade de grupo tutorial no aprendizado da disciplina de imunologia no módulo de imunopatologia sob a perspectiva dos alunos do $3^{\circ}$ semestre do curso de medicina da UFC - campus Cariri. Anais... IV Encontro Universitário da UFC no Cariri Juazeiro do Norte-CE. 
Pereira, A. S., et al. (2018). Metodologia da pesquisa científica. [e-book]. Santa Maria. Ed. UAB/NTE/UFSM. Disponível em: https://repositorio.ufsm.br/bitstream/ handle/1/15824/Lic_Computacao_Metodologia-Pesquisa-Cientifica.pdf?sequence=1 .

Pimpão, C. T., Rocha, R. M. V. M., Chi, K. D., Teixeira, V. N., Madeira, H. M. F., Michelotto, A. L., Guebert, M. C. C., \& Burchard, J. (2017). Desenvolvendo competências na aprendizagem de estudantes de medicina veterinária. Veterinária e Zootecnia, 24 (3), 592603.

Lanne, E. (2008). Problem-Based Learning in Veterinary Education. Journal of Veterinary Medical Education, 35 (4): 631- 636.

Lucena, R. M. S., Souza, S. R., Campos, A. F. (2012). Concepções Alternativas dos Alunos Iniciantes do Curso de Medicina Veterinária sobre Reações Químicas: contextos de uma investigação. Acta Scientiae, 14 (3), 472-487.

Saliba, N. A. A., Moimaz, S. A. S., Chiaratto, R. A., \& Tiano, A. V. P. (2008). A utilização da metodologia PBL em Odontologia: descortinando novas possibilidades ao processo ensinoaprendizagem. Revista Odontologia Ciência, 23 (4),392-396.

\section{Porcentagem de contribuição de cada autor no manuscrito}

Renata Ferreira dos Santos - $40 \%$

Jordana Belos dos Santos - $30 \%$

Cristina Alves Moreira - $30 \%$ 